\title{
LexFindR: A fast, simple, and extensible R package for finding similar words in a lexicon
}

\author{
ZhaoBin $\mathrm{Li}^{1}$, Anne Marie Crinnion ${ }^{2,3}$, \& James S. Magnuson ${ }^{2,3}$ \\ ${ }^{1}$ Department of Mathematics and Statistics, Carleton College, Northfield, MN, USA \\ ${ }^{2}$ Institute for the Brain and Cognitive Sciences, University of Connecticut, Storrs, CT, USA \\ ${ }^{3}$ Department of Psychological Sciences, University of Connecticut, Storrs, CT, USA
}

\begin{abstract}
Language scientists often need to generate lists of words and potential competitors. They may do this for purposes of experimental control (e.g., selecting items matched on lexical neighborhood but varying in word frequency), or to test theoretical predictions (e.g., hypothesizing that a novel type of competitor may impact word recognition). Several online tools are available, but most are constrained to a fixed lexicon and set of competitor definitions, and may not give the user full access or control of source data. We present LexFindR, an open source $\mathrm{R}$ package that can be easily modified to include additional, novel competitor types. LexFindR is easy to use. Because it can leverage multiple CPU cores and uses vectorized code, it is also extremely fast. In this article, we present an overview of LexFindR usage, illustrated with examples, explain the details of how we implemented several standard lexical competitor types used in spoken word recognition research (e.g., cohorts, neighbors, embeddings, rhymes), and show how "lexical dimensions" (e.g., word frequency, word length, uniqueness point) can be integrated into LexFindR workflows (for example, to calculate "frequency weighted competitor probabilities").
\end{abstract}

Keywords: psycholinguistics; lexicon; word recognition 


\section{Introduction}

Language scientists often need to generate sets of related words or words with specific properties. This might be in service of experimental control (e.g., words matched on length and frequency of occurrences, but differing in lexical neighborhood; Luce \& Pisoni, 1998). Or the need might arise based on a theoretically-motivated or model-driven hypothesis; perhaps your theory proposes - or your model simulations predict - that shorter words embedded within a word should make that word more difficult to process, so you want to find words with many or few words embedded within them. Sets of related items and their characteristics can also be useful for clinical purposes. For example, frequency-weighted lexical neighborhoods have proven useful for clinical assessments and interventions (e.g., Kirk, Pisoni, \& Osberger, 1995; Morrisette \& Gierut, 2002; Sommers \& Danielson, 1999; Storkel, Bontempo, Aschenbrenner, Maekawa, \& Lee, 2013; Storkel, Maekawa, \& Hoover, 2010). So how do we generate these lists?

Various excellent tools already exist. For example, three web-based tools are Michael Vitevtich's phonotactic probability (Vitevitch \& Luce, 1998, 1999) and neighborhood density calculators (http: //www.people.ku.edu/ mvitevit/PhonoProbHome.html), the English Lexicon Project (https: //elexicon.wustl.edu/; Balota et al., 2007), and the recent Auditory English Lexicon Project (https://inetapps.nus.edu.sg/aelp; Goh, Yap, \& Chee, 2020). Other tools exist for semantic variables or languages other than English, such as Lexique, which includes English and French (http: //www.lexique.org/; New, Pallier, Brysbaert, \& Ferrand, 2004) and the multilingual CLEARPOND (https://clearpond.northwestern.edu/; Marian, Bartolotti, Chabal, \& Shook, 2012), but it takes considerable independent work for a researcher to combine these resources with things like neighborhood statistics from the other tools.

Furthermore, while these tools are incredibly useful, they have limitations. Many require using web interfaces, so a researcher's workflow must include interacting with the websites and documenting the steps taken, and importing lists of items into the researcher's local workflow (e.g., into R; R Core Team, 2019). One might argue that this is not a major inconvenience, but other limitations are more severe. For example, so far as we are aware, the computer code used to search lexicons on the sites listed above are not readily available, so a researcher can neither easily confirm the code's validity or extend it (for example, to include a new type of potential competitor). Another limitation is that some tools have a predefined lexicon, and a researcher cannot substitute another in its place. Substituting your own lexicon might be useful if you simply prefer a different lexicon, or if you were using an artificial lexicon, either with human subjects or with a computational model, or if you wanted to examine an understudied language or dialect. Finally, we assume that many labs and researchers have developed and will continue to develop their own code for lexical searches. This duplication of effort is unfortunate. An open-source, extensible tool shared via a version-control repository would allow researchers to collaborate and share their extensions, reducing duplication of effort.

We have developed a lightweight $\mathrm{R}$ package, LexFindR (Li, Crinnion, \& Magnuson, 2020), that addresses these limitations. LexFindR comes with a suite of lexical relation finders for common competitor types (e.g., neighbors, cohort [onset] competitors, and rhymes), but is also easily extended to incorporate new definitions. LexFindR is also fast, as it uses R's parallelization capabilities to leverage multiple CPU cores (typically found even on contemporary laptops) and uses vectorized code where possible (e.g., using R's apply family of functions). Appendix 1 provides an example of how to put together aspects of the examples throughout the paper in order to efficiently gather information about multiple lexical dimensions in one script. In the following sections, we review how to install and use LexFindR. Details about how to share extensions with the community via LexFindR's github repository are provided in Appendix 2. 


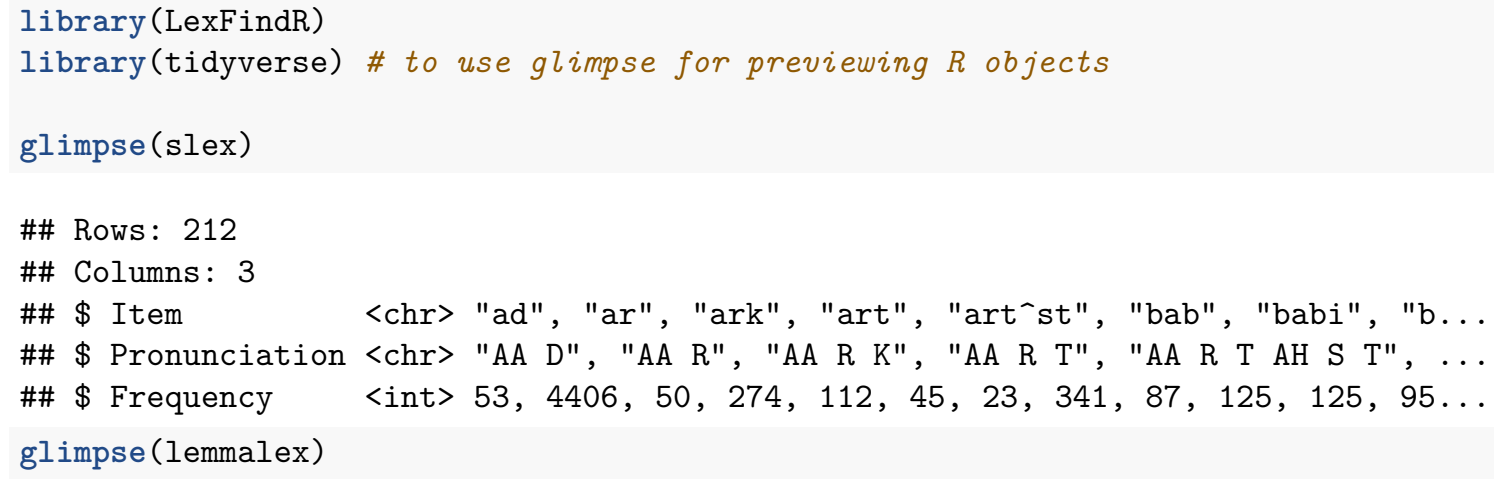

library (LexFindR)

\section{Getting started}

The package comes with two lexicons: the 212-word slex lexicon (with only 14 phonemes) from the TRACE model of spoken word recognition (McClelland \& Elman, 1986) as a small data set for the user to experiment with, and a larger lexicon (lemmalex) that we compiled from various open-access, non-copyrighted materials. The primary source is the SUBTLEX subtitle corpus (Brysbaert \& New, 2009), which we cross-referenced with the copyrighted Francis and Kučera (1982) database to reduce the word list to "lemma" (base- or uninflected forms). Pronunciations were drawn from the larger CMU Pronouncing Dictionary (CMU Computer Science, 2020) without lexical stress for both lexicons (with those for slex transcribed by Nenadić and Tucker (2020)). The second lexicon is large enough to demonstrate the full capabilities of the package. The two data sets are automatically loaded when we load LexFindR. We use the tidyverse (Wickham et al., 2019) glimpse function to view essential details about the lexicons, and view their first few lines. 


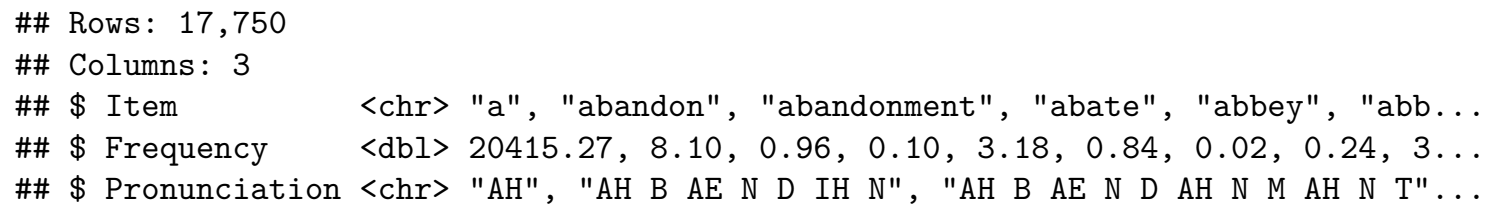

Both lexicons are loaded as $\mathrm{R}$ dataframes with three fields. "Item" is a label (orthography in the case of lemmalex, and transcriptions in the original phonemic conventions used for the TRACE model in the case of slex). Pronunciation is a space-delimited phonemic transcription using the ARPAbet conventions of the CMU Pronouncing Dictionary (ARPAbet transcriptions for TRACE items are from Nenadić \& Tucker, 2020). "Frequency" is occurrences-per-million words; frequencies are based on Kučera and Francis (1967) for slex and on Brysbaert and New (2009) for lemmalex.

More information about the lexicons can by queried with the '?' command:

?slex

?lemmalex

120

Note that you can use any lexicon you can load into an $\mathrm{R}$ dataframe. You may find it convenient to use the same field names as in slex and lemmalex, but it is not necessary. For work on phonological word forms, you typically will have both "Item" (usually orthography) and "Pronunciation", but as we will see later, you can do useful things with LexFindR with any list of forms, including orthographic forms.

\section{LexFindR commands}

To use any of the LexFindR functions, we provide a target pattern and a word list to compare it to. LexFindR will compare the target pattern to the patterns in the word list to find items that have particular relations to the target. The functions can return indices of items that meet the criteria of the function, but we can also tell LexFindR to return instead the list of matching forms, the list of accompanying labels for matching forms (e.g., spellings), or the frequencies of matching forms. As we progress through examples, we will see when these different options are useful.

Cohorts. Let's begin with cohorts. Cohorts are words that overlap at word onset, and are called "cohorts" because they comprise the set of words predicted to be strongly activated as a spoken word is heard (and thus to form the recognition cohort) by the Cohort Model (Marslen-Wilson \& Welsh, 1978). While definitions vary, LexFindR uses a very common cohort definition: overlap in the first two phonemes. We can get the set of cohort indices for a pattern with a command like this for the pronunciation of CAR:

get_cohorts("K AA R", slex\$Pronunciation)

\#\# [1] $66 \begin{array}{lllll}67 & 68 & 69 & 70 & 71\end{array}$

This tells us that slex entries 66-71 are cohorts of CAR. To get the competitors themselves rather than the indices, we could specify that we want forms:

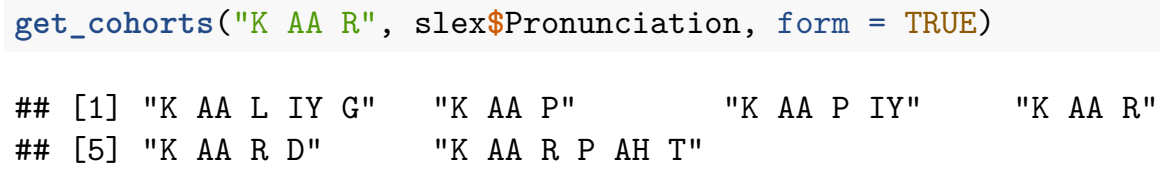


To see the labels of those items (in TRACE's phonemic transcriptions), we can use standard $\mathrm{R}$ conventions (and should see the phonemic transcriptions for COLLEAGUE, COP, COPY, CAR, CARD, and CARPET):

slex[get_cohorts("K AA R", slex\$Pronunciation), ]\$Item

\#\# [1] "kalig" "kap" "kapi" "kar" "kard" "karp`t"

Alternatively, we could request the count of cohorts:

get_cohorts("K AA R", slex\$Pronunciation, count = TRUE)

\#\# [1] 6

That is not a large number of cohorts. Let's compare it to the count we get from lemmalex:

get_cohorts("K AA R", lemmalex\$Pronunciation, count = TRUE)

\#\# [1] 272

As expected, we get many more from a more realistically-sized lexicon. Note that most LexFindR functions have exactly the same structure, returning indices by default, but with options to return forms or counts.

Neighborhood. Neighbors are another possible competitor often considered in spoken word recognition research. The standard neighbor definition comes from the Neighborhood Activation Model (NAM; Luce \& Pisoni, 1998). While NAM includes a graded similarity rule, most often, researchers use the simpler DAS rule: two words are considered neighbors (and are expected to be strongly activated if either one is heard) if they differ by no more than a single phonemic deletion, $a$ ddition, or substitution. For example, CAR (/kar/) has many neighbors, including the deletion neighbor ARE (note that neighbors are based on pronunciation here, not spelling), addition neighbors SCAR and CARD, and substitution neighbors at every position, such as BAR, CORE, and COP (though as we will see, there CAR has no medial [vowel] substitution neighbors in slex). Let's look at CAR's neighbors in slex, using analogous commands to those we used for cohorts.

\# get indices

get_neighbors("K AA R", slex\$Pronunciation)

\#\# [1] $\quad 2 \quad \begin{array}{llllllll}\text { \#\# } & 10 & 67 & 69 & 70 & 104 & 152 & 184\end{array}$

\# get forms

get_neighbors("K AA R", slex\$Pronunciation, form = TRUE)

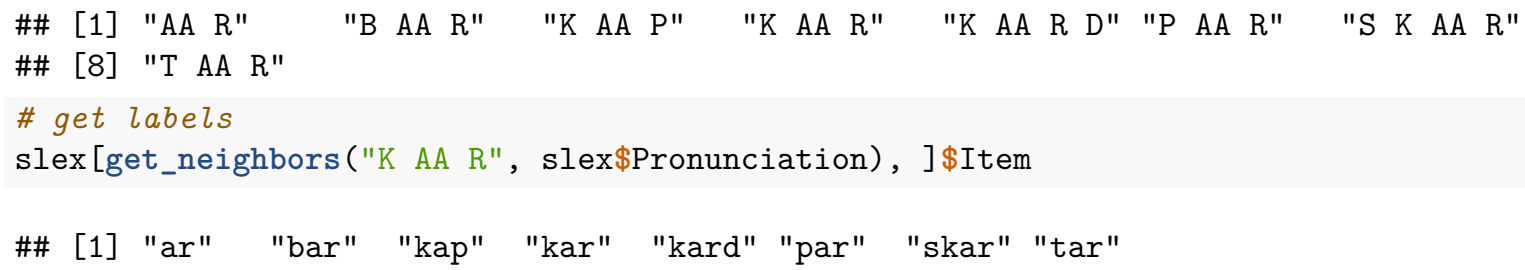




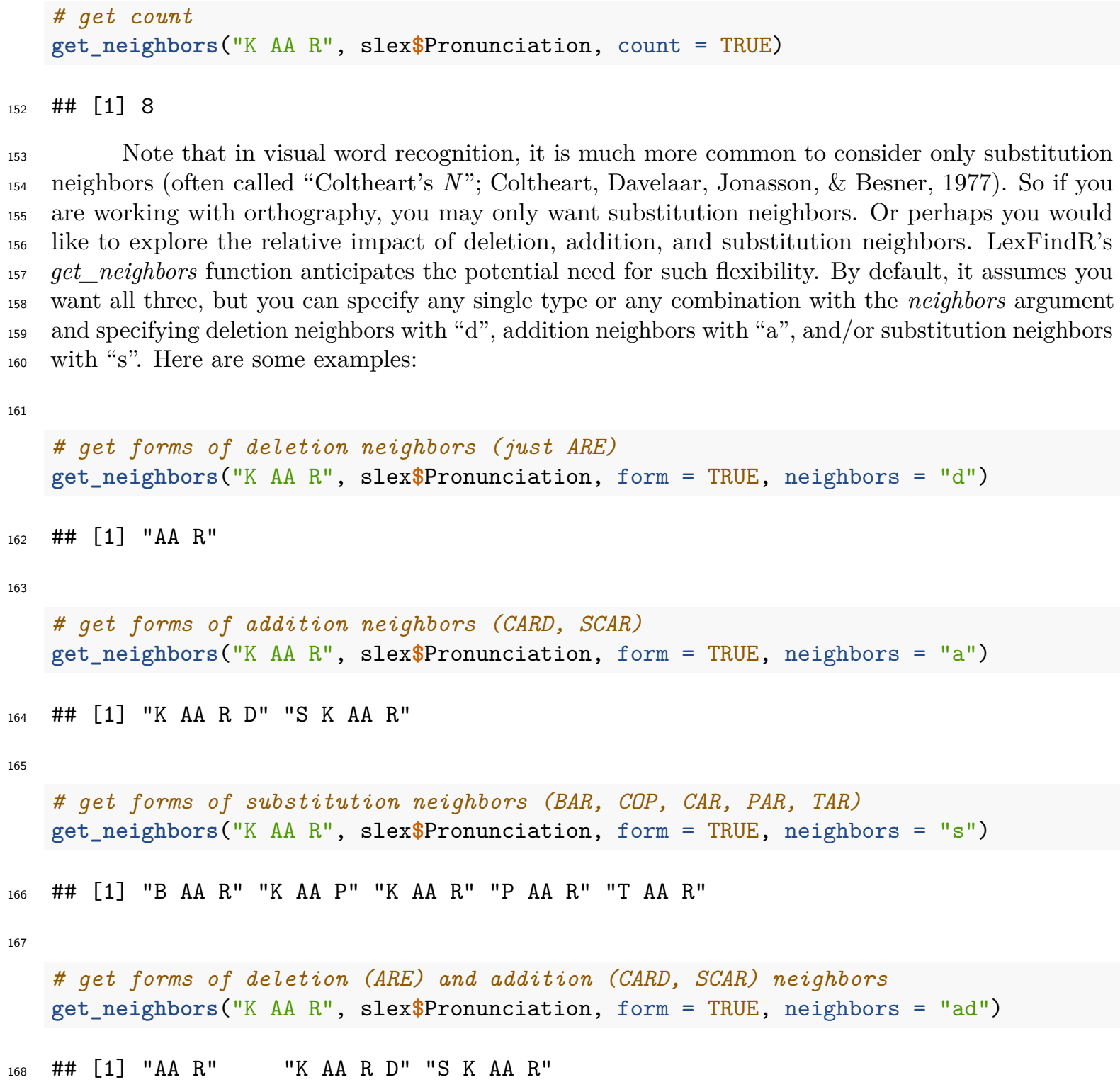

\#\# [1] 8

Note that in visual word recognition, it is much more common to consider only substitution neighbors (often called "Coltheart's N"; Coltheart, Davelaar, Jonasson, \& Besner, 1977). So if you are working with orthography, you may only want substitution neighbors. Or perhaps you would like to explore the relative impact of deletion, addition, and substitution neighbors. LexFindR's get_neighbors function anticipates the potential need for such flexibility. By default, it assumes you want all three, but you can specify any single type or any combination with the neighbors argument and specifying deletion neighbors with "d", addition neighbors with "a", and/or substitution neighbors with "s". Here are some examples:

\# get forms of deletion neighbors (just ARE)

get_neighbors("K AA R", slex\$Pronunciation, form = TRUE, neighbors = "d")

\#\# [1] "AA R"

\# get forms of addition neighbors (CARD, SCAR)

get_neighbors("K AA R", slex\$Pronunciation, form = TRUE, neighbors = "a")

\#\# [1] "K AA R D" "S K AA R"

\# get forms of substitution neighbors (BAR, COP, CAR, PAR, TAR)

get_neighbors("K AA R", slex\$Pronunciation, form = TRUE, neighbors = "s")

\#\# [1] "B AA R" "K AA P" "K AA R" "P AA R" "T AA R"

\# get forms of deletion (ARE) and addition (CARD, SCAR) neighbors

get_neighbors("K AA R", slex\$Pronunciation, form = TRUE, neighbors = "ad")

\#\# [1] "AA R" "K AA R D" "S K AA R"

Of course, we can easily do other things using basic R commands, such as determine what proportion of CAR's neighbors are substitution neighbors:

\# what proportion of CAR's neighbors are substitution neighbors?

get_neighbors("K AA R", slex\$Pronunciation, neighbors = "s", count = TRUE) /

get_neighbors("K AA R", slex\$Pronunciation, count = TRUE)

\#\# [1] 0.625

Other competitor types. In addition to cohorts and neighbors, LexFindR comes with analogous functions for several other similarity types.

- get_rhymes: returns items that mismatch at word onset by a maximum of 1 phoneme (so not a standard definition of poetic rhyme or phonological rime). Rhymes will include items that 
are addition or deletion neighbors at first position (e.g., CAR's rhymes will include ARE and SCAR) as well as substitution neighbors at position 1 (e.g., BAR, TAR).

- get_embeds_in target: returns items that are embedded within a target word. For SCAR, this would include ARE and CAR.

- get_target_embeds_in: returns items that the target embeds within. For CAR, this would include SCAR and CARD.

- get_homoforms: returns items with the same form as the target. We use "homoform" because these would be homophones for phonological forms but homonyms for orthographic forms.

LexFindR also anticipates the possibility that a researcher may want to find competitor types that do not overlap. For example, CARD is both a cohort and a neighbor of CAR, so which set should it appear in? We propose a novel category called nohorts - neighbors that are also cohorts and provide "P" (pure) versions of several competitor-type functions that return non-overlapping sets.

- get_nohorts: Cohorts and neighbors are overlapping sets, although not all cohorts are neighbors (e.g., CAR and CARPET are cohorts but not neighbors) and not all neighbors are cohorts. Nohorts are the intersection of cohorts and neighbors. Note that the target word will be part of the nohort set, and not part of cohortsP or neighborsP, which we define next.

- get_cohortsP: the set of "pure" cohorts that are not also neighbors.

- get_neighborsP: the set of "pure" neighbors that are not also cohorts or rhymes.

- get_embeds_in_targetP: set of items that embed in the target that are not also cohorts or neighbors.

- get_target_embeds_inP: set of items that the target embeds in that are not also cohorts or neighbors.

The nohort and "P" functions use the base-R intersect and setdiff functions to find set intersections and differences. To see the code for any function in R, you can simply enter the function name with no arguments and no following parentheses. Let's look at the code for get_nohorts. Many of the details provided may not be useful for a typical user, but the intersect command is the interesting part of this example.

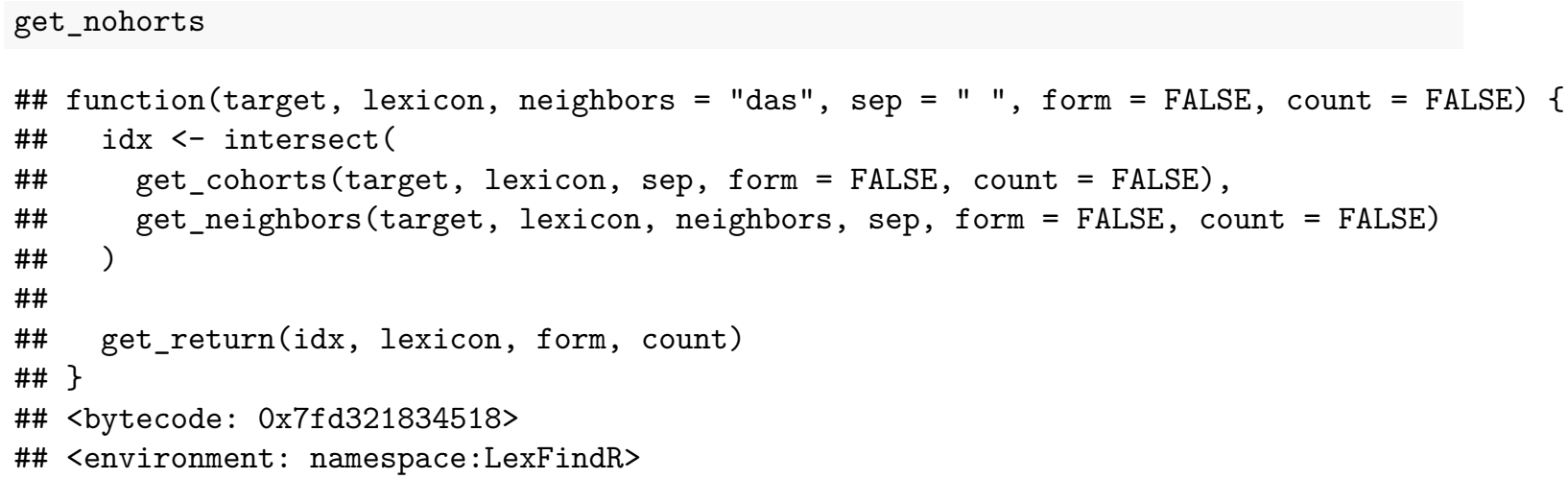

Note that if you just wanted to look at neighbors, cohorts, and nohorts - but not rhymes - you would want to add the list of rhymes (obtained with get_rhymes) to the list returned by get_neighborsP.

Form length. You may wish to calculate form length. This is easy to do with base R. If you use CMU pronunciations, as in lemmalex, we can use a technique for counting words separated by whitespace with the lengths command in R. 
223

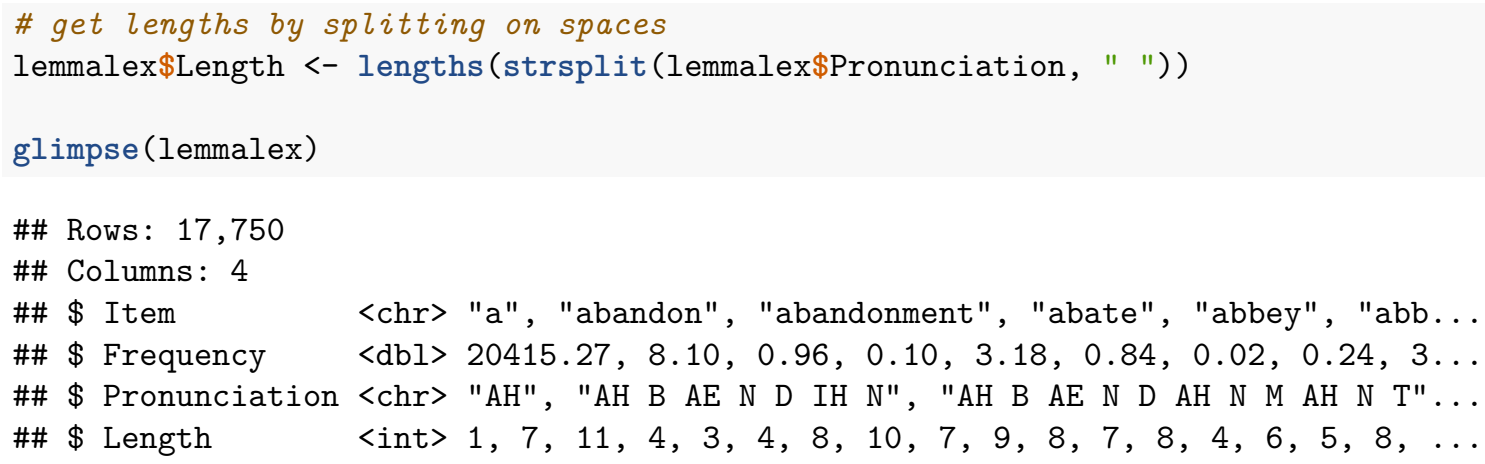

If you have a null-delimited form, where each character is a single letter or phoneme, we can use the nchar function.

\# get lengths by counting characters for orthography or 1-char per phoneme forms slex\$Length <- nchar (slex\$Item)

glimpse (slex)

\#\# Rows: 212

\#\# Columns: 4

\#\# \$ Item <chr> "ad", "ar", "ark", "art", "art`st", "bab", "babi", "b... \#\# \$ Pronunciation <chr> "AA D", "AA R", "AA R K", "AA R T", "AA R T AH S T", .. \#\# \$ Frequency <int> 53, 4406, 50, 274, 112, 45, 23, 341, 87, 125, 125, 95... \#\# \$ Length $\quad$ <int> 2, 2, 3, 3, 6, 3, 4, 4, 4, 3, 4, 5, 2, 4, 3, 4, 3, 4,..

Uniqueness point. We have added one other common lexical dimension to the LexFindR functions (get_uniqpt), which is the uniqueness point (UP) of a form. This is the position at which an item becomes the only completion in the lexicon. For example, in slex, /kard/ (CARD) becomes unique at position 4, as does /karp^t/ (CARPET). SCAR becomes unique at position 3. CAR $(/ \mathrm{kar} /)$ is not unique at its final position, so its uniqueness point is set to its length plus one.

get_uniqpt("K AA R", slex\$Pronunciation)

\#\# [1] 4

get_uniqpt("S K AA R", slex\$Pronunciation)

\#\# [1] 3

Again, CAR is not unique by word offset, so its UP is its length plus one. SCAR becomes unique at position 3, one before its offset. Let's consider some additional useful steps. We could normalize UPs by dividing them by word length plus one, the maximal possible score. So CARD would have a normalized UP of $0.8(4 / 5)$, while CARPET's would be $0.57(4 / 7)$, and CAR's would be $1.0(4 / 4)$. Here are some examples.

\# Get UPs for all items in slex

slex\$UP <- unlist (lapply (slex\$Pronunciation, FUN = get_uniqpt, lexicon = slex\$Pronunciation 


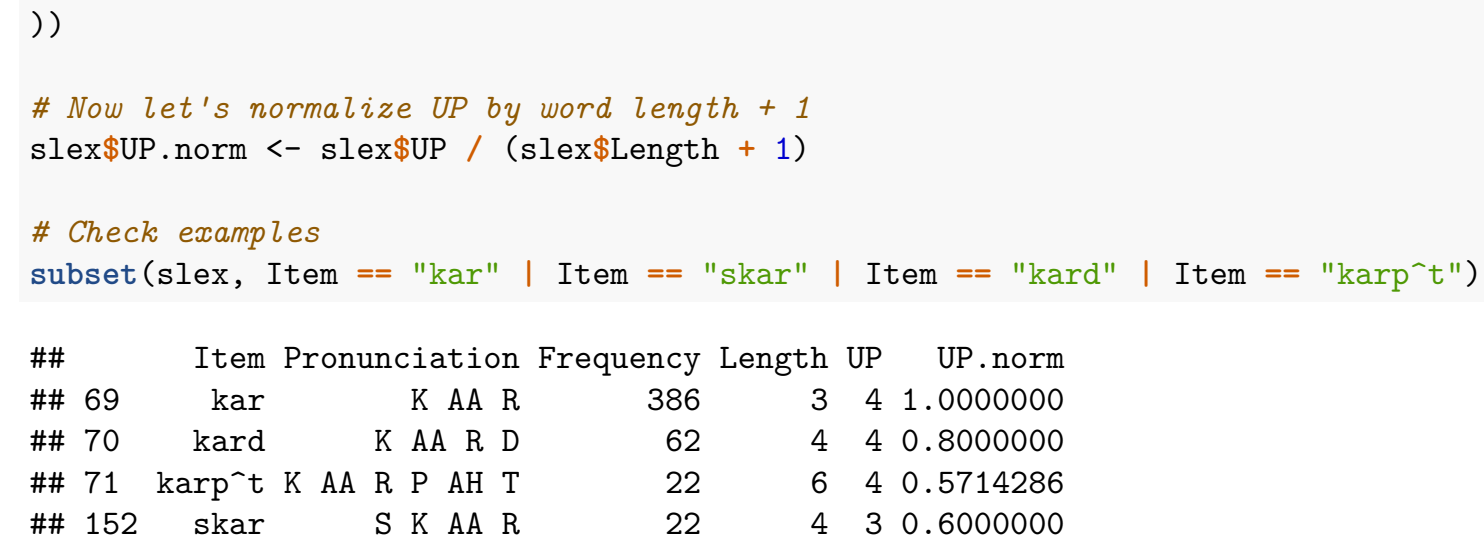

\section{Helper functions}

\# get CAR's lemmalex cohorts' frequencies

llex_cohort_frequencies <- lemmalex\$Frequency [

] get_neighbors("K AA R", lemmalex\$Pronunciation)

summary (llex_cohort_frequencies)

Typically, frequencies are log scaled, as this provides a better fit when they are used to predict human behavior (e.g., word recognition time). It would be useful, therefore, to weight the count of competitors by log frequencies. The LexFindR helper function get $f w$ does this. You supply it with a list of frequencies, and it takes their logs and returns the sum. This is simple enough that you could do it with basic $\mathrm{R}$ functions yourself. However, get fw provides some useful error checking. Specifically, it checks whether the minimum frequency in your set of frequencies is less than one, since taking the log would return a negative value. If so, it also suggests a minimum constant to specify for pad to add to each frequency before taking the log. Let's consider how we might use this. First, let's try using get fw to give us summed log frequencies for the frequencies we collected above for CAR's slex cohorts. 
get_fw(slex_cohort_frequencies)

\#\# [1] 35.1571

This gives us the sum without any problem, as the minimum frequency in slex_cohort_frequencies is greater than 1. Now let's try with llex_cohort_frequencies.

get_fw(llex_cohort_frequencies)

\#\# Warning: 'min(competitors_freq) + pad' is 0.22 which is $<1$;

\#\# * Consider adding pad >= 0.78

\#\# [1] 55.64038

Now we get a value (55.64038) but also a warning because the minimum value is less than 1 . So let's add the pad option. Using 1 will bring our minimum to a value greater than 1 , avoiding results with non-positive values.

get_fw(llex_cohort_frequencies, pad = 1)

\#\# [1] 65.67193

Log Frequency Weighted Competitor Probabilities: get fwcp. We could go a step beyond frequency weights and calculate the Frequency Weighted Competitor Probability (FWCP) of a word, inspired by the Neighborhood Activation Model's Frequency-Weighted Neighborhood Probability (FWNP; Luce \& Pisoni, 1998). This is calculated as the ratio of the target word's log frequency to the sum of all words meeting the competitor definition, as in the following equation.

$$
F W C P=\frac{\log \left(\text { Frequency }_{\text {target }}\right)}{\sum_{c \in \text { competitors }} \log \left(\text { Frequency }_{c}\right)}
$$

Notably, on most competitor definitions, this includes the target word itself, so we can think of the ratio as expressing what proportion of the "frequency weight" of the target's competitors is contributed by the target itself. For spoken words, the larger the ratio, the more easily the target word tends to be recognized. To calculate this with LexFindR, we supply a set of competitor frequencies and the target word's frequency to the get fwcp function. Note that we can include a pad option as for get fw, and it will be applied to both the target word's frequency and the list of competitor frequencies; again, this should be done if the minimum frequency value is less than 1 . Let's verify that the minimum frequency in slex is greater than 1.

\# check the minimum frequency

$\min (\mathrm{slex} \$ F r e q u e n c y)$

\#\# [1] 10

The next two code blocks demonstrate how to get the FWCP for neighbors (i.e., the FWNP) and then for cohorts.

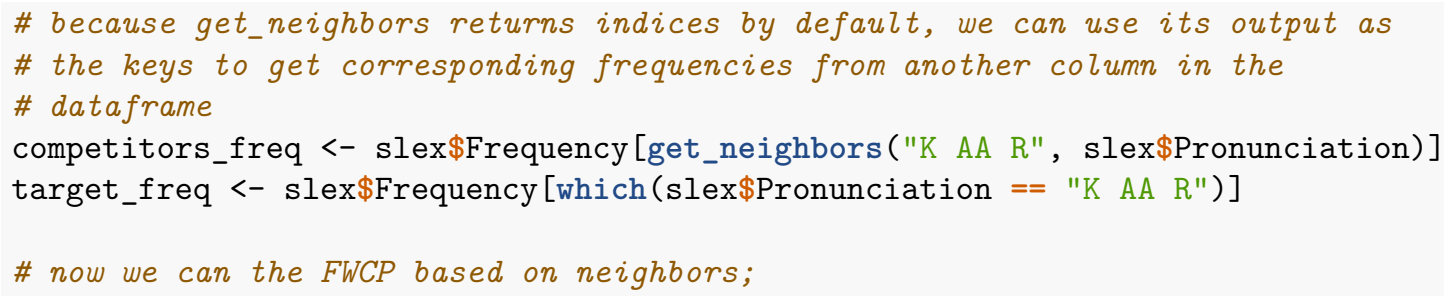




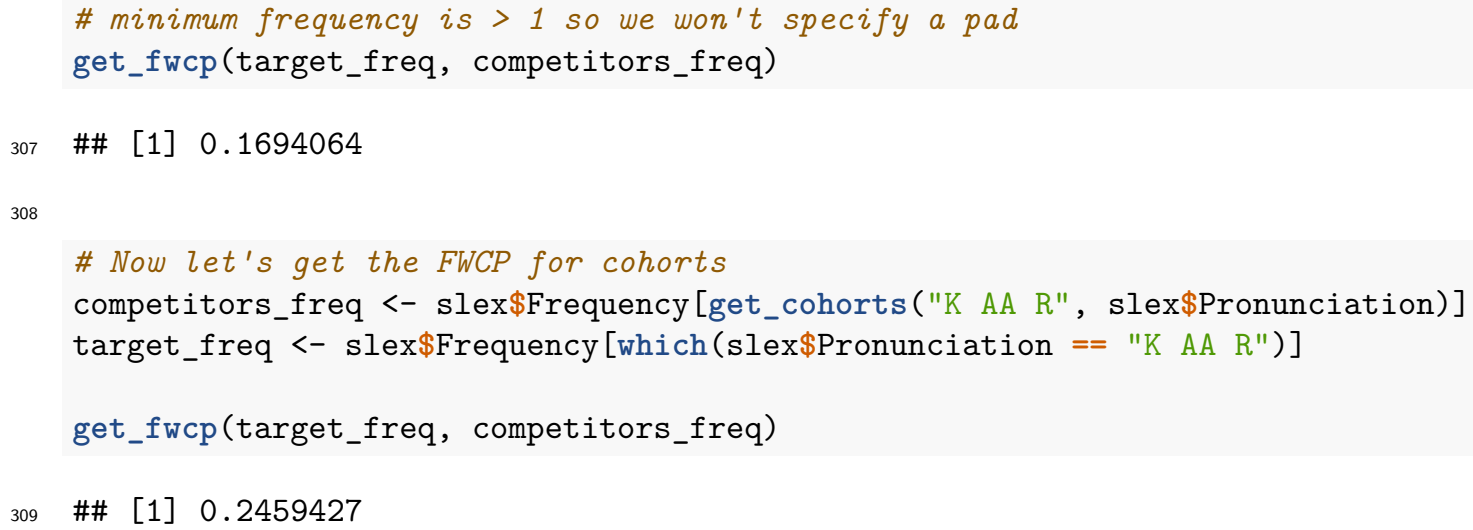

\#\# [1] 0.1694064

Note that get fwcp is not simply computing the ratio of target-to-competitor frequencies; it is first converting the frequencies to log frequencies. If your lexicon file has frequencies already in log form, you should not use the get fwcp function, but instead you should calculate the ratios directly. Also note that it is fairly standard to express frequencies as occurrences-per-million. If your basis is different (e.g., occurrences-per-six million), you may want to transform your frequencies to the more standard per-million basis. Finally, we recommend that you examine distributions before using the results of get fwcp, as these often exhibit difficult-to-mitigate deviations from normality. One may be better served by examining target frequencies and competitor frequency weights (obtained with get_fw) separately.

\section{Working with orthography or other "undelimited" forms, or other delimiters}

By default, LexFindR functions expect the forms you supply to be space-delimited, which is the typical convention for CMU pronunciations. Using a delimiter allows you to have form codes (typically phoneme codes) made up of more than one character. But what if you want to work with orthography, or a phoneme code that uses one character per phoneme without delimiters? You can simply specify sep $="$ " to indicate that your forms have a "null" delimiter. We can illustrate this with the orthography in the "Item" field in lemmalex.

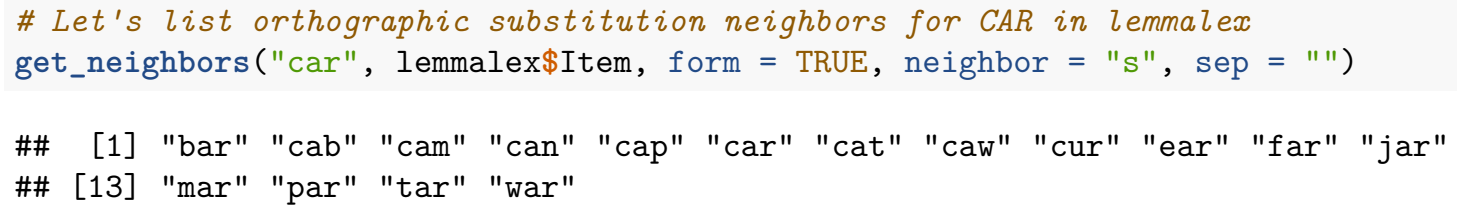

Now let's try it with TRACE's original phoneme encodings, which use one character per phoneme. Those original forms are in the "Item" field of slex:

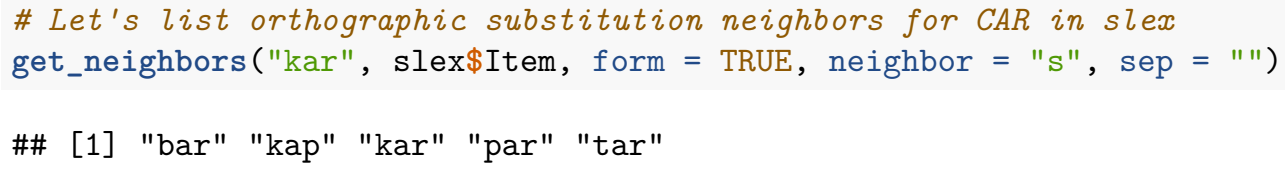

\section{Batch processing with target list and lexicon}

Often, we may need to get the competitors for each word in the lexicon, with respect to the entire lexicon. This would be a prerequisite for selecting words with relatively many vs. few neighbors, 
336

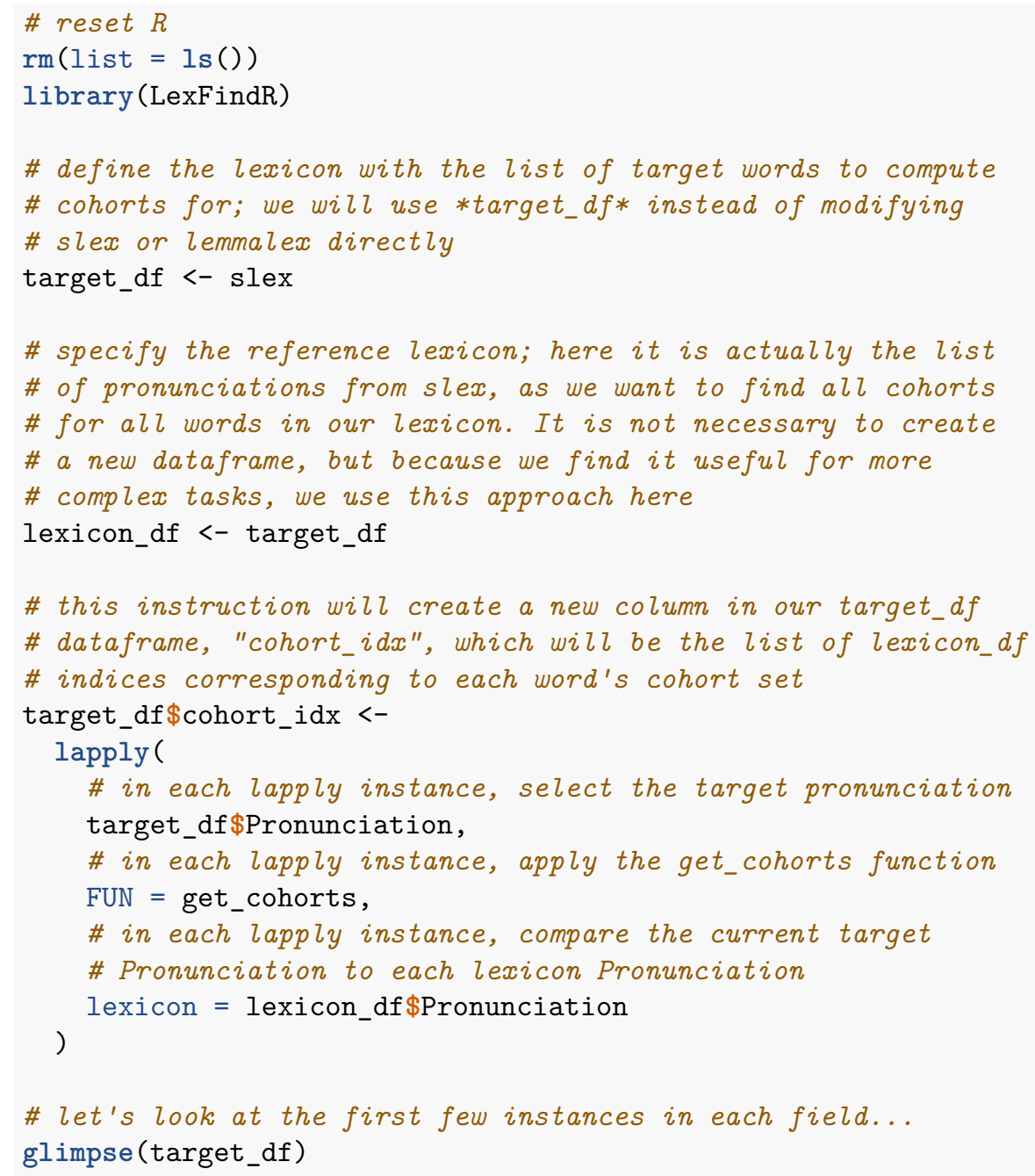

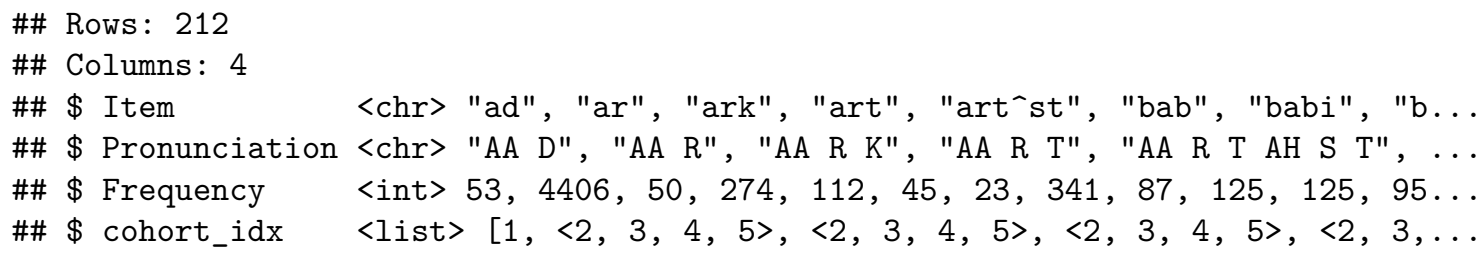

Consider the cohort_idx field. We can see that/ad/ (ODD) has only one cohort (itself), while /ar/ (ARE) has four (items 2, 3, 4, 5, or /ar/, /ark/,/art/, and /art^st/, i.e., ARE, ARK, ART, ARTIST).

What if we also want the lists of cohort forms or labels and frequencies? Rather than calling the function three times, we could speed up the process (speed will be very important when we work with large lexicons!) by calling get_cohorts only once, and then using the indices to get the other items we want. In the next example, we keep working with target_ $d f$ and its new field cohort_idx (which has the list of indices [row counts] of records that meet the cohort definition for each target). 
352

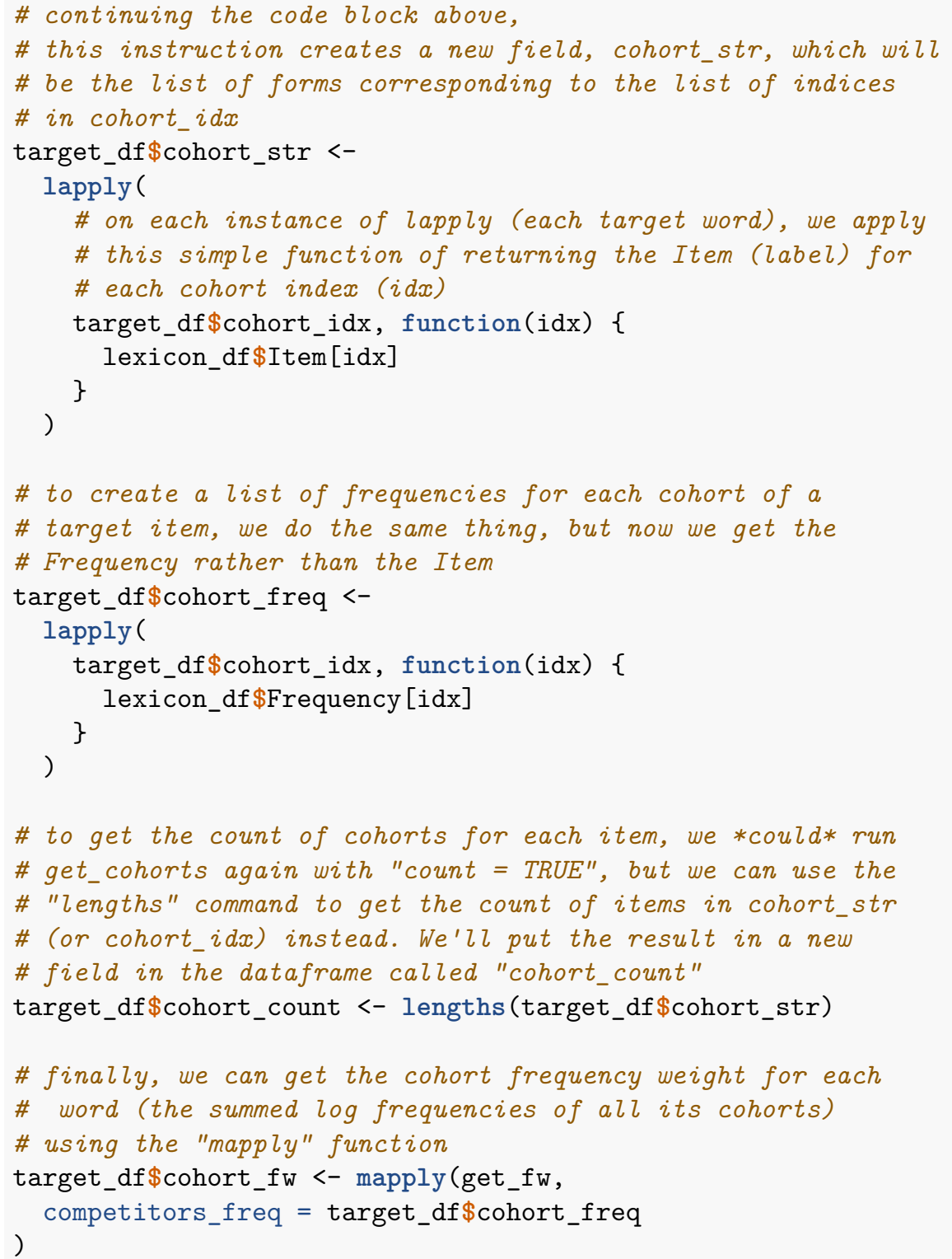

353

Let's look at the results:

glimpse(target_df)

\#\# Rows: 212

\#\# Columns: 8

\#\# \$ Item <chr> "ad", "ar", "ark", "art", "art^st", "bab", "babi", "b... \#\# \$ Pronunciation <chr> "AA D", "AA R", "AA R K", "AA R T", "AA R T AH S T", ... \#\# \$ Frequency <int> 53, 4406, 50, 274, 112, 45, 23, 341, 87, 125, 125, 95...

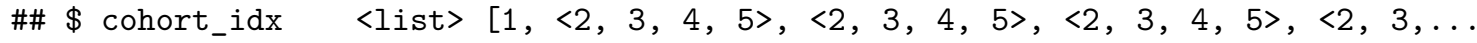
\#\# \$ cohort_str <list> ["ad", <"ar", "ark", "art", "art`st">, <"ar", "ark",... 


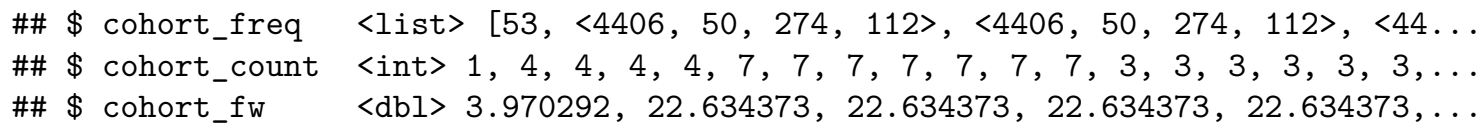

We can see that cohort_idx, cohort_str, and cohort_freq all contain lists, and we can verify that for a given word, the lists are the same length (e.g., one frequency form for each cohort). There should only be one value per target word in cohort_count and cohort_fw, which we can see is the case as well.

Working with different target and lexicon lists. In some cases, you may only want to get details for a subset of items in the lexicon - or even for a list of forms that are not in the lexicon. In these cases, you can simply specify a shorter target list rather than making the target list and lexicon the same. Note that of course, if you do not have frequencies for your items, you will not be able to use the get fwcp command. As an example, we might want to examine what the neighborhoods of the words in the TRACE lexicon would be in the context of a realistically-sized lexicon. We can do this by using slex as our target list and lemmalex as our lexicon.

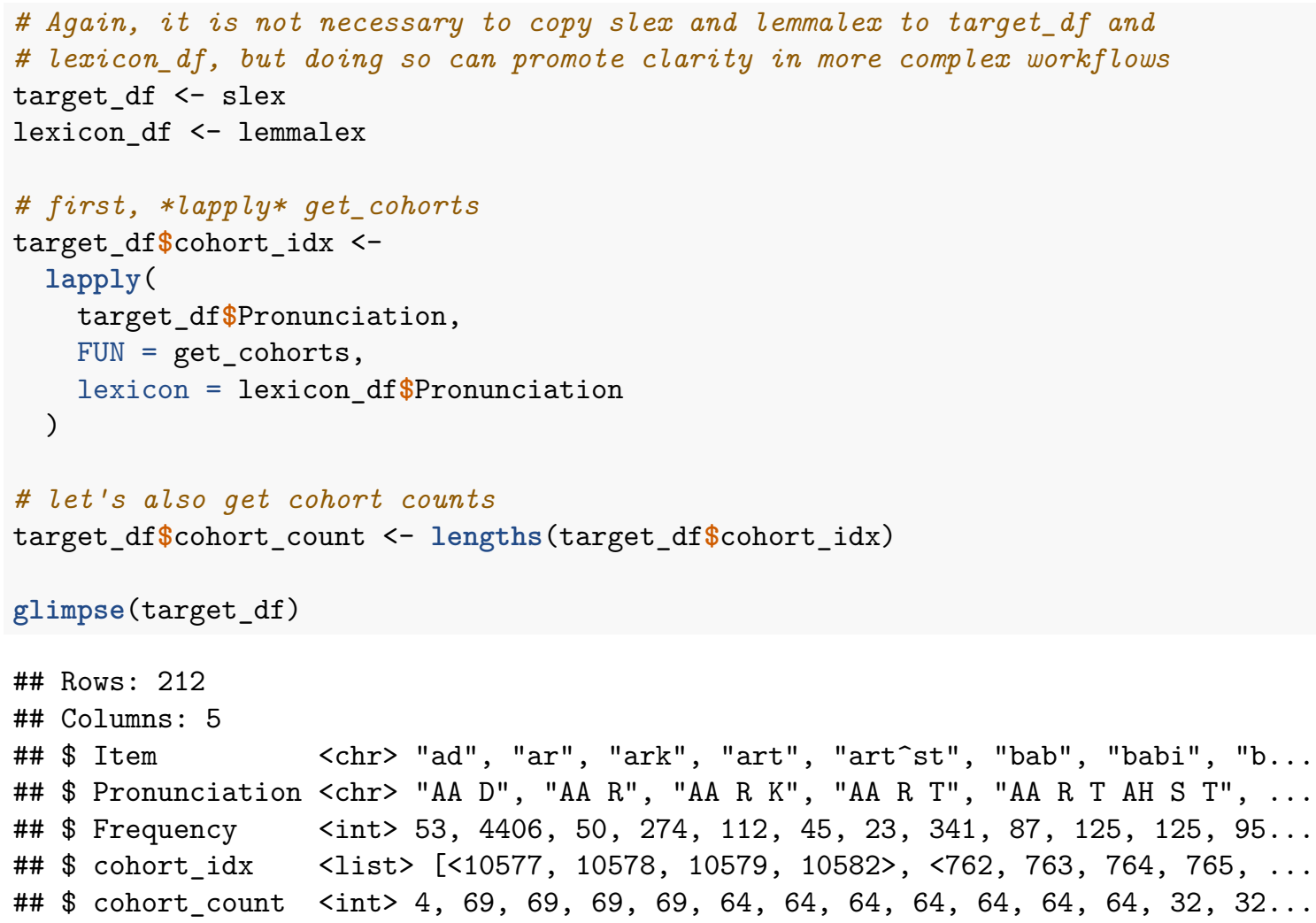

Comparing this to our earlier results, we see that ODD would have 12 cohorts in lemmalex instead of 1 within slex.

\section{Parallelizing for speed}

If we are getting competitors for every word in a lexicon, speed becomes a concern, especially if we want to do this for many competitor types. To quantify this, let's time how long it takes to calculate cohorts for all cohorts in lemmalex. We will use the $\mathrm{R}$ tictoc package (Izrailev, 2014) to time the process. For this demonstration, we are using a MacBook Pro with an Intel Core i9 CPU and $32 \mathrm{gb}$ of RAM. 


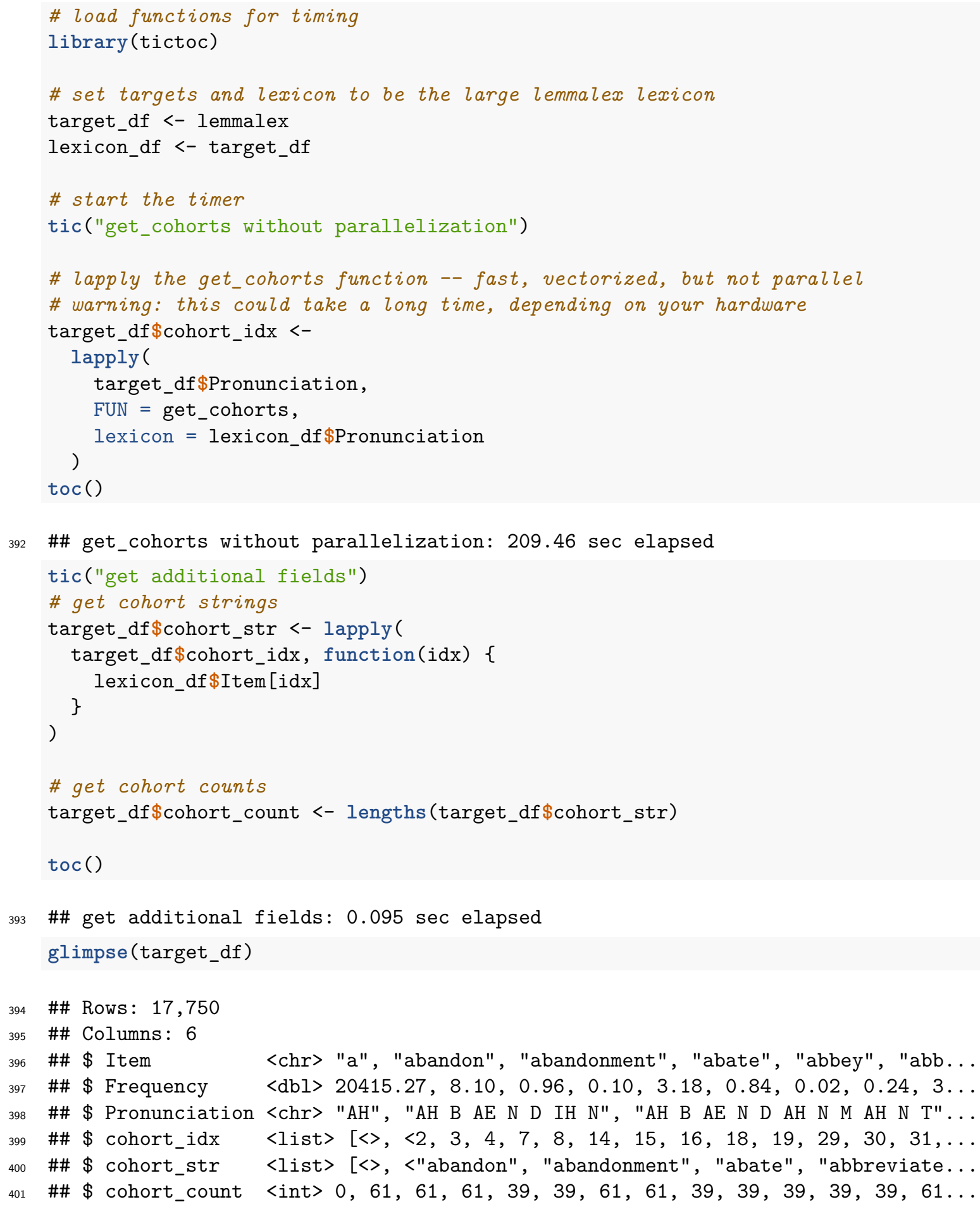

On our demonstration laptop, get_cohorts with lapply took 133 seconds (on a Linux workstation we tested, it took nearly 15 minutes). If you only have to do this once, that may be tolerable. But we can do better! We could easily parallelize using the $\mathrm{R}$ future package, and its commands like 
406

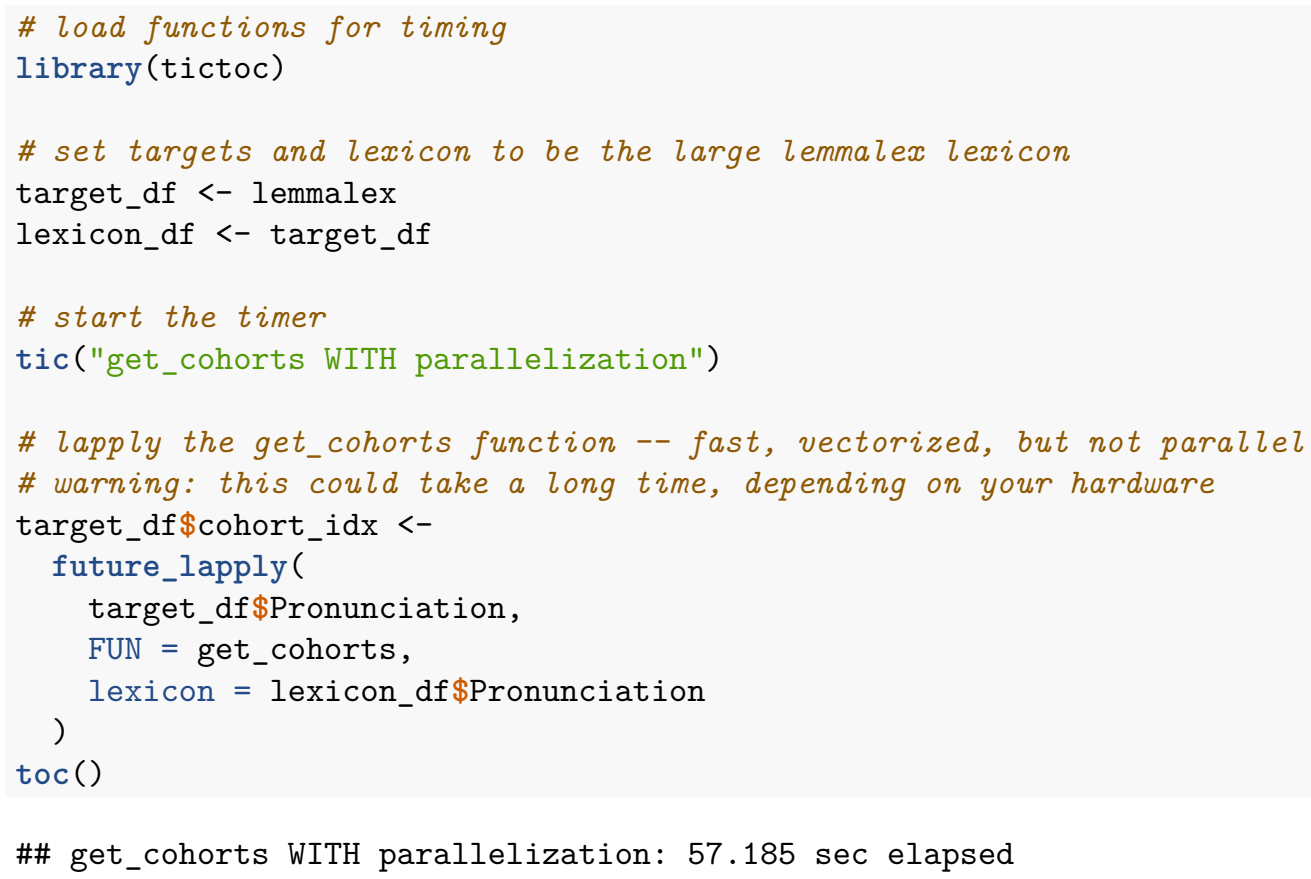




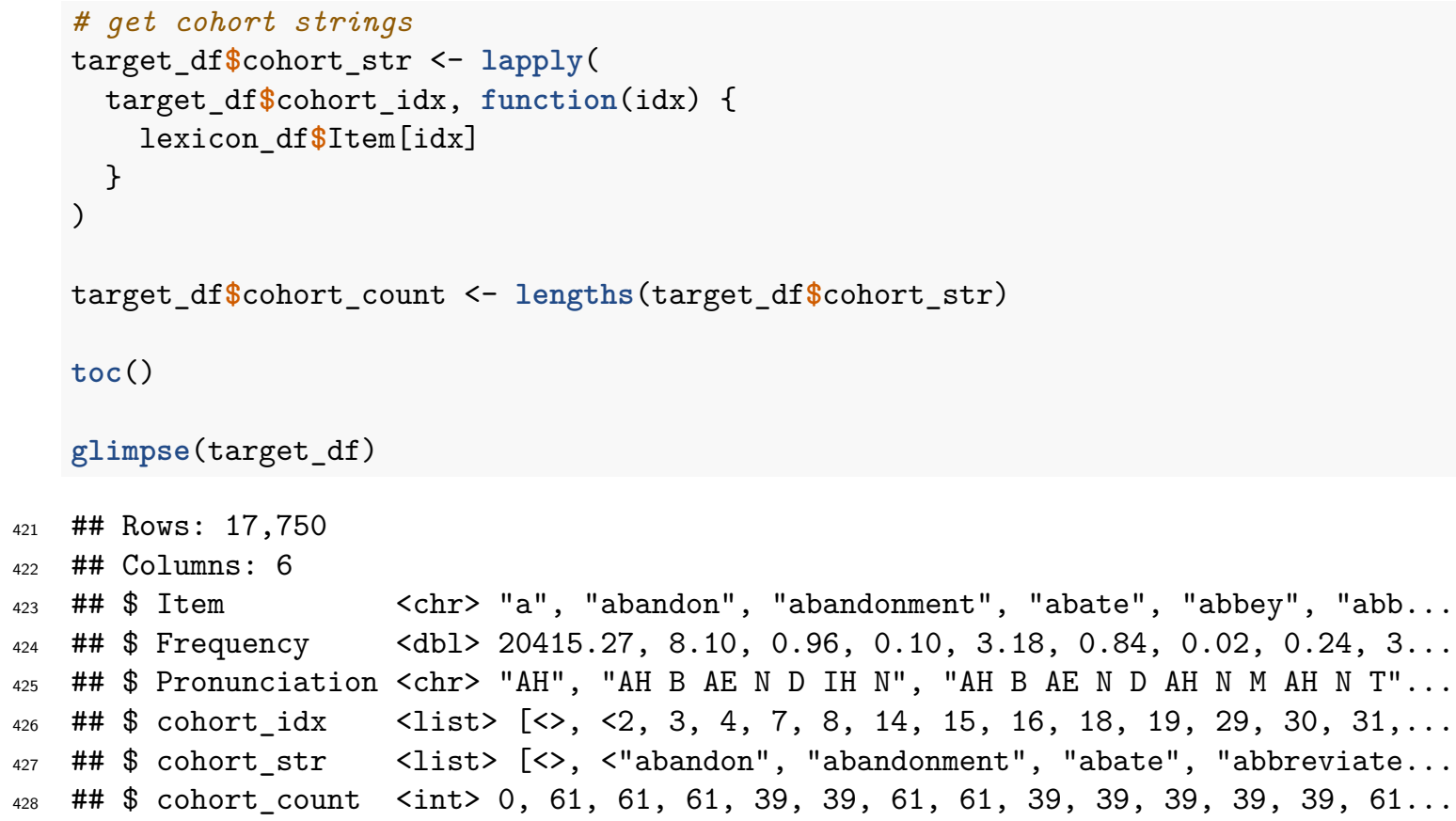

We see an improvement from 133 seconds to approximately 31; it took a bit more than 4 times longer without parallelization. On the Linux workstation, the improvement was more dramatic, from $\sim 14$ minutes to $\sim 30$ seconds ( $\sim 28$ times faster with parallelization). Again, such differences may not seem important if you are running a search once, but if you want to do many different kinds of searches, or explore novel similarity definitions, speed will become important. In Appendix 1, we present an example of parallelized code for conducting several LexFindR competitor searches in 435 series.

\section{Conclusions}

LexFindR fills important gaps in the language scientist's toolkit. It provides a free, fast, extensible, tested, and readily shared tool that can be integrated into typical analysis workflow within R. Researchers inclined to contribute extensions to LexFindR should refer to Appendix 2 for basic guidance on how to do so. We hope our fellow researchers will find LexFindR useful. 
441

\section{Author contributions}

ZL and JM conceptualized the project; ZL wrote most code and drafted most of this manuscript; AMC contributed significant documentation to the LexFindR package and contributed to the writing and editing of the full manuscript; JM advised on and contributed to code and writing, and contributed to and edited the full manuscript.

\section{Acknowledgments}

This work was supported, in part, by U.S. National Science Foundation grants PAC 1754284 (JM, PI) and IGE NRT 1747486 (JM, PI). The authors are solely responsible for the content of this article. 


\section{Appendix 1: Extended example - Getting several competitor types}

This example shows how you can go through several competitor types for a lexicon, adding columns for the indices, labels, frequencies, counts, frequency weights, and FWCP for each competitor type. For an example implemented in tidyverse piping style, see the package vignettes for LexFindR.

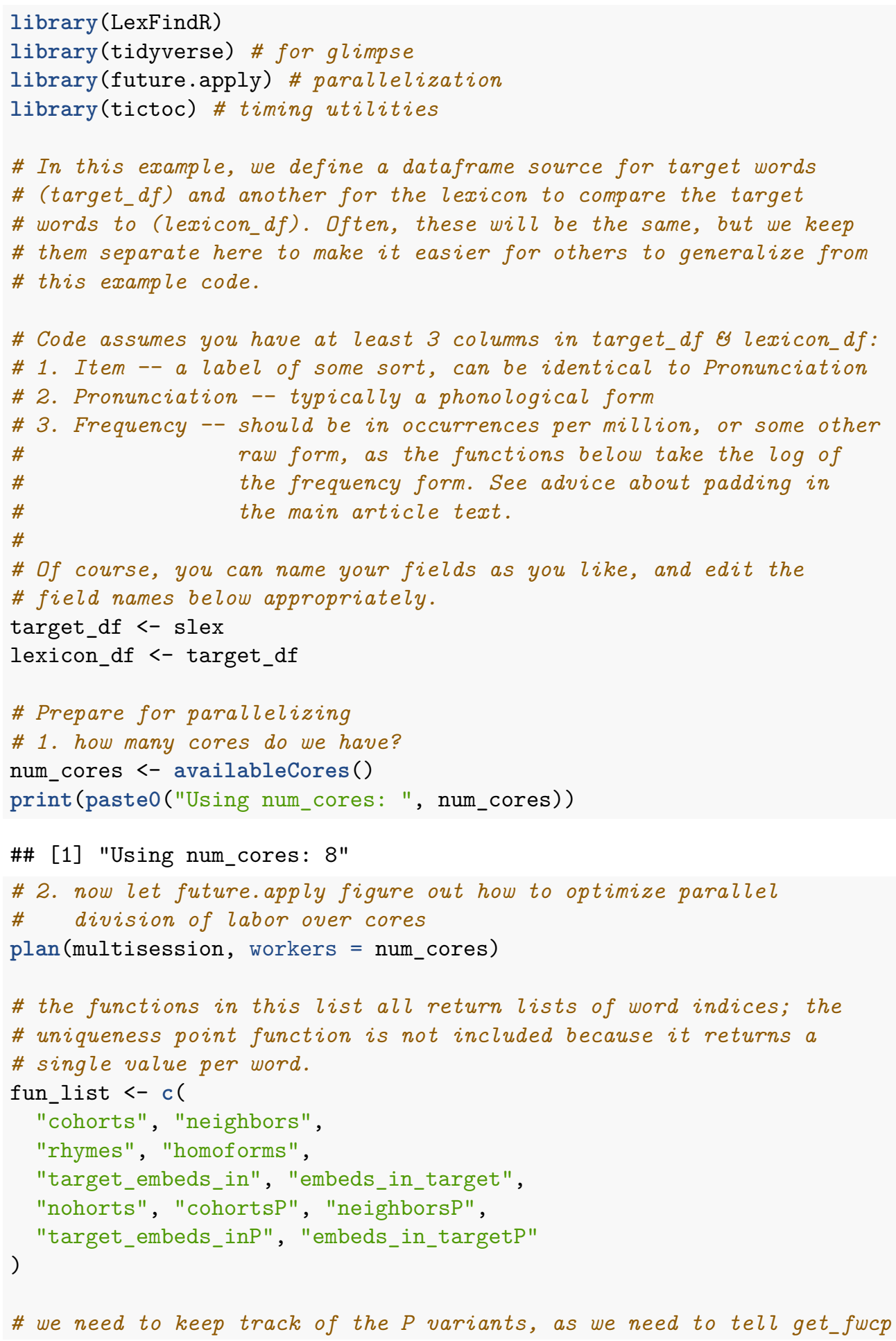




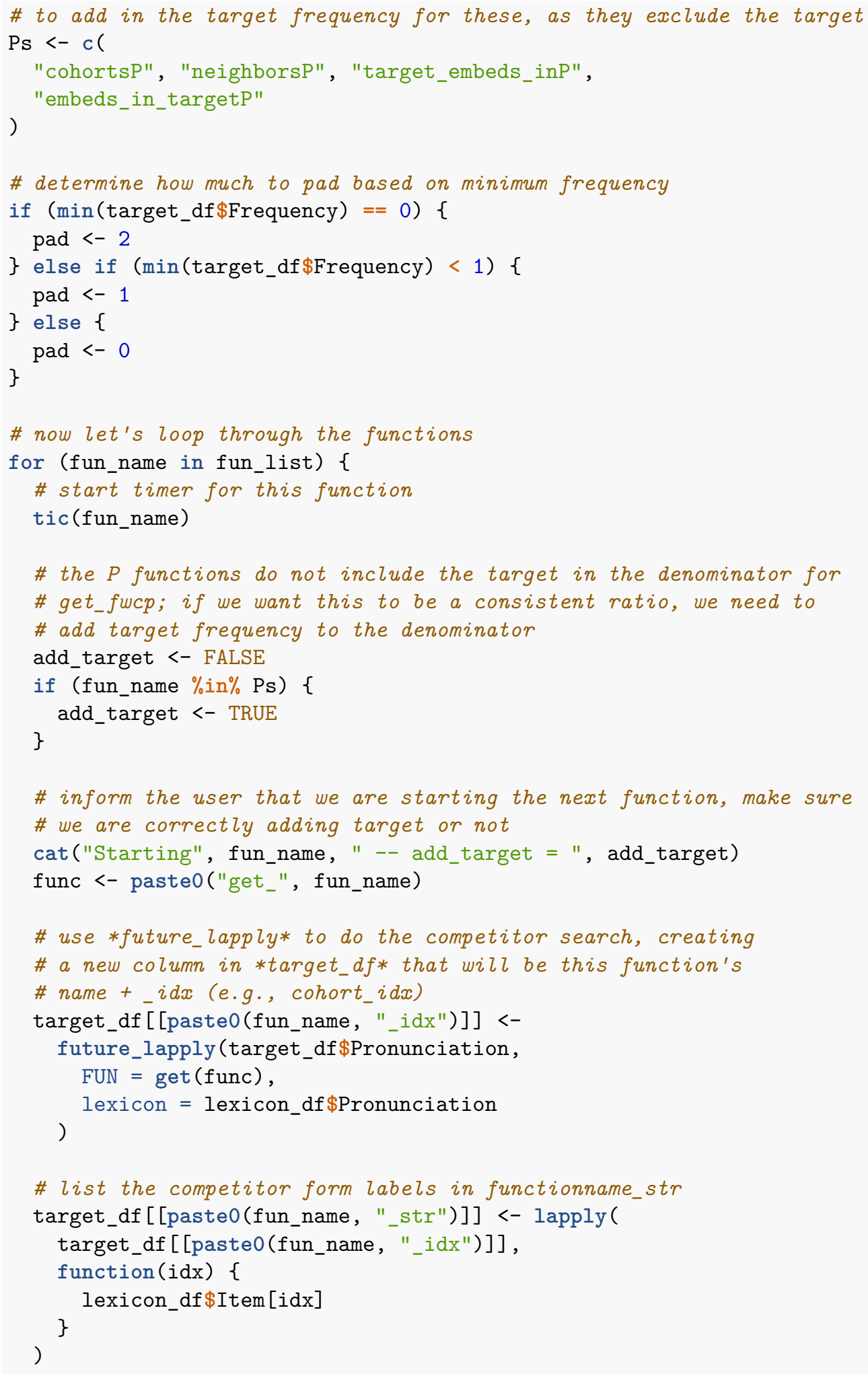




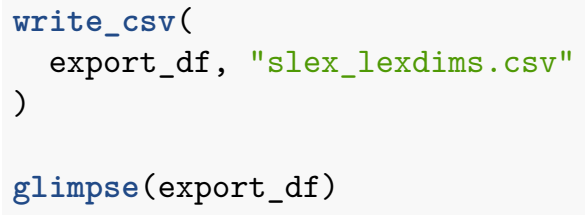

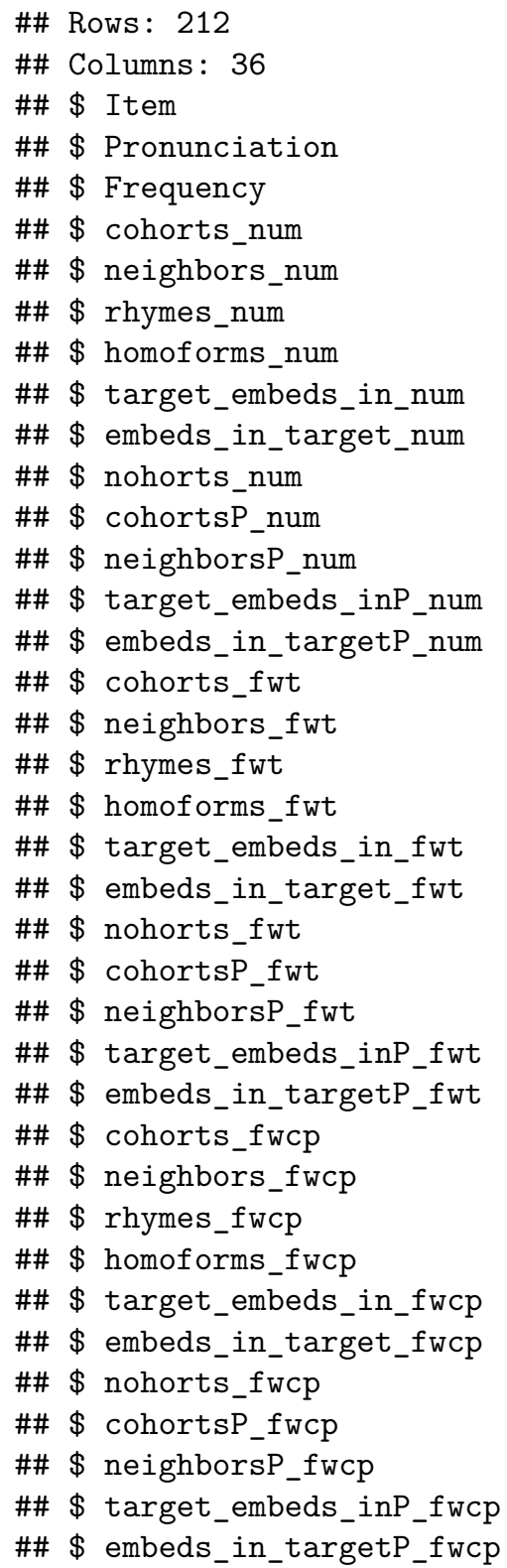

<chr> "ad", "ar", "ark", "art", "artst", "bab", "... <chr> "AA D", "AA R", "AA R K", "AA R T", "AA R $T$.. <int> 53, 4406, 50, 274, 112, 45, 23, 341, 87, 125... <int> $1,4,4,4,4,7,7,7,7,7,7,7,3,3,3, \ldots$ <int> $4,8,6,5,1,4,4,2,1,7,5,1,7,5,8, \ldots$ <int> $3,5,4,3,1,2,2,1,1,5,4,1,6,3,4, \ldots$ <int> $1,1,1,1,1,1,1,1,1,1,1,1,1,1,1, \ldots$ <int> $6,29,5,9,1,2,1,1,1,2,1,1,5,1,1 \ldots$ <int> $1,1,2,2,5,1,3,2,1,2,4,2,1,3,3, \ldots$ <int> $1,3,3,3,1,3,3,2,1,3,2,1,2,2,3, \ldots$ <int> $0,1,1,1,3,4,4,5,6,4,5,6,1,1,0, \ldots$ <int> $1,1,0,0,0,0,0,0,0,0,0,0,0,1,2, \ldots$ <int> $3,21,1,5,0,0,0,0,0,0,0,0,2,0,0 \ldots$ <int> $0,0,0,0,2,0,1,1,0,0,1,1,0,0,0, \ldots$ $\langle\mathrm{dbl}\rangle$ 3.970292, 22.634373, 22.634373, 22.634373, 2... $\langle\mathrm{dbl}\rangle 21.533445,37.968634,33.688446,27.349358, \ldots$ $\langle\mathrm{dbl}\rangle$ 13.142723, 24.473191, 19.684596, 15.046612, ..

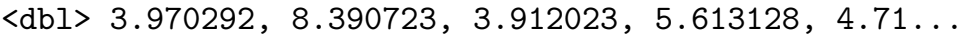
$\langle\mathrm{dbl}>29.792782,127.685319,22.680328,42.517044, \ldots$ $\langle\mathrm{dbl}\rangle$ 3.970292, 8.390723, 12.302746, 14.003851, 35... $\langle\mathrm{dbl}>3.970292,17.915874,17.915874,17.915874,4 \ldots$ $\langle\mathrm{dbl}>0.000000,4.718499,4.718499,4.718499,17.9 \ldots$ $\langle\mathrm{dbl}\rangle$ 8.390723, 3.970292, 0.000000, 0.000000, 0.00... $\langle\mathrm{dbl}\rangle$ 16.650059, 88.968478, 2.995732, 22.751933, 0.. $\langle\mathrm{dbl}\rangle 0.000000,0.000000,0.000000,0.000000,16.5 \ldots$ $\langle\mathrm{dbl}\rangle 1.00000000,0.37070710,0.17283550,0.247991 \ldots$ $\langle\mathrm{dbl}\rangle 0.1843779,0.2209909,0.1161236,0.2052380, \ldots$ $\langle\mathrm{dbl}\rangle 0.3020905,0.3428536,0.1987352,0.3730493, \ldots$ $\langle\mathrm{db} 1\rangle 1,1,1,1,1,1,1,1,1,1,1,1,1,1,1, \ldots$ $\langle\mathrm{dbl}\rangle 0.13326355,0.06571407,0.17248529,0.132020 \ldots$ $\langle\mathrm{dbl}\rangle 1.0000000,1.0000000,0.3179797,0.4008275, \ldots$ $\langle\mathrm{dbl}\rangle 1.0000000,0.4683401,0.2183551,0.3133047, \ldots$ $\langle\mathrm{dbl}\rangle 1.0000000,0.6400626,0.4532777,0.5432957, \ldots$ $\langle\mathrm{dbl}\rangle 0.3211947,0.6788053,1.0000000,1.0000000, \ldots$ $\langle\mathrm{dbl}>0.19254240,0.08618315,0.56632333,0.197888 \ldots$ $\langle\mathrm{dbl}\rangle 1.0000000,1.0000000,1.0000000,1.0000000, \ldots$ 


\section{Appendix 2: Bug reports and user contributions}

How to report bugs. Report any bugs at https://github.com/maglab-uconn/LexFindR/i ssues by clicking on "New Issue".

How to create an extension. To contribute new functions, first please read the $\mathrm{R}$ files that are part of the LexFindR package. New functions can be added to extensions. $R$ on your local installation. New functions should be carefully tested and the code should be clearly commented. Once you are confident your code is ready to be shared, move on to the next step of submitting your code via github.

How to contribute extensions via github. Extensions are welcomed through a github "pull request". Once the user has created a local clone of the forked repository, the user can edit the competitors. $R$ or extensions. $R$ file and push their edits to their forked path. Once these edits have been made, users can open a pull request. Before every pull request, run R CMD check to ensure that the code is clean. Please also style your code using the tidyverse style guide at https://style.tidyverse.org/ (Wickham, n.d.) and document your code using roxygen2 (Wickham, Danenberg, Csárdi, \& Eugster, 2020). We will monitor pull requests and merge appropriate changes. 


\section{References}

Balota, D. A., Yap, M. J., Cortese, M. J., Hutchison, K. A., Kessler, B., Loftis, B., ... Treiman, R. (2007). The english lexicon project. Behavior Research Methods, 39, 445-459. https: //doi.org/10.3758/BF03193014

Bengtsson, H. (2013). future: Unified Parallel and Distributed Processing in $R$ for Everyone.

Brysbaert, M., \& New, B. (2009). Moving beyond Kučera and Francis: A critical evaluation of current word frequency norms and the introduction of a new and improved word frequency measure for American English. Behavior Research Methods, 41, 977-990.

CMU Computer Science. (2020). CMU Pronouncing Dictionary. Pittsburgh, PA, USA: Carnegie Mellon University. Retrieved from http://www.speech.cs.cmu.edu/cgi-bin/cmudict,\%20Aug ust $\% 2025, \% 202020$.

Coltheart, M., Davelaar, E., Jonasson, J. T., \& Besner, D. (1977). Access to the internal lexicon. In S. Dornic (Ed.) (pp. 535-555). Hillsdale, NJ, USA: Erlbaum.

Francis, W., \& Kučera, H. (1982). Frequency Analysis of English usage: Lexicon and Grammar. Boston: Houghton Mifflin.

Goh, W. D., Yap, M. J., \& Chee, Q. W. (2020). The Auditory English Lexicon Project: A multi-talker, multi-region psycholinguistic database of 10,170 spoken words and nonwords. Behavior Research Methods. https://doi.org/10.3758/s13428-020-01352-0

Izrailev, S. (2014). tictoc: Functions for timing $R$ scripts, as well as implementations of Stack and List structures.

Kirk, K. I., Pisoni, D. B., \& Osberger, M. J. (1995). Lexical effects on spoken word recognition by pediatric cochlear implant users. Ear and Hearing, 16, 470-481. https://doi.org/10.1097/00 003446-199510000-00004

Kučera, H., \& Francis, W. (1967). Computational Analysis of Present-Day American English. Providence, RI, USA: Brown University Press.

Li, Z., Crinnion, A. M., \& Magnuson, J. S. (2020). LexFindR: Find related items and lexical dimensions in a lexicon. Retrieved from https://github.com/maglab-uconn/LexFindR

Luce, P. A., \& Pisoni, D. B. (1998). Recognizing spoken words: The neighborhood activation model. Ear and Hearing, 19, 1-36. https://doi.org/10.1097/00003446-199802000-00001

Marian, V., Bartolotti, J., Chabal, S., \& Shook, A. (2012). CLEARPOND: Cross-linguistic easyaccess resource for phonological and orthographic neighborhood densities. PLoS ONE, 7 . https://doi.org/10.1371/journal.pone.0043230

Marslen-Wilson, W. D., \& Welsh, A. (1978). Processing interactions and lexical access during word recognition in continuous speech. Cognitive Psychology, 10, 29-63. https://doi.org/https: //doi.org/10.1016/0010-0285(78)90018-X

McClelland, J. L., \& Elman, J. L. (1986). The TRACE model of speech perception. Cognitive Psychology, 18, 1-86.

Morrisette, M. L., \& Gierut, J. A. (2002). Lexical organization and phonological change in treatment. Journal of Speech, Language, and Hearing Research, 45, 143-159. https://doi.org/10.1044/ 1092-4388(2002/011)

Nenadić, F., \& Tucker, B. V. (2020). Computational modelling of an auditory lexical decision experiment using jTRACE and tisk. Language, Cognition and Neuroscience, 1-29. 
Nenadić, F., \& Tucker, B. V. (2020). Computational modelling of an auditory lexical decision experiment using jTRACE and TISK. Language, Cognition and Neuroscience, 0, 1-29. https://doi.org/10.1080/23273798.2020.1764600

New, B., Pallier, C., Brysbaert, M., \& Ferrand, L. (2004). Lexique 2: A new french lexical database. Behavior Research Methods, Instruments, and Computers, 36, 516-524. https: //doi.org/10.3758/BF03195598

R Core Team. (2019). R: A language and environment for statistical computing. Vienna, Austria: R Foundation for Statistical Computing. Retrieved from https://www.R-project.org/

Sommers, M. S., \& Danielson, S. M. (1999). Inhibitory processes and spoken word recognition in young and older adults: The interaction of lexical competition and semantic context. Psychology and Aging, 14, 458-472. https://doi.org/10.1037/0882-7974.14.3.458

Storkel, H. L., Bontempo, D. E., Aschenbrenner, A. J., Maekawa, J., \& Lee, S.-Y. (2013). The effect of incremental changes in phonotactic probability and neighborhood density on word learning by preschool children. Journal of Speech, Language, and Hearing Research, 56, 1689-1700. https://doi.org/10.1044/1092-4388(2013/12-0245)

Storkel, H. L., Maekawa, J., \& Hoover, J. R. (2010). Differentiating the effects of phonotactic probability and neighborhood density on vocabulary comprehension and production: A comparison of preschool children with versus without phonological delays. Journal of Speech, Language, and Hearing Research, 53, 933-949. https://doi.org/10.1044/1092-4388(2009/090075)

Vitevitch, M. S., \& Luce, P. A. (1998). When words compete: Levels of processing in perception of spoken words. Psychological Science, 9, 325-329. https://doi.org/10.1111/1467-9280.00064

Vitevitch, M. S., \& Luce, P. A. (1999). Probabilistic phonotactics and neighborhood activation in spoken word recognition. Journal of Memory and Language, 40, 374-408. https://doi.org/ 10.1006/jmla.1998.2618

Wickham, H. (n.d.). The tidyverse style guide. Retrieved from https://style.tidyverse.org/

Wickham, H., Averick, M., Bryan, J., Chang, W., McGowan, L. D., François, R., ... Yutani, H. (2019). Welcome to the tidyverse. Journal of Open Source Software, 4(43), 1686. https://doi.org/10.21105/joss.01686

Wickham, H., Danenberg, P., Csárdi, G., \& Eugster, M. (2020). Roxygen2: In-line documentation for $r$. Retrieved from https://CRAN.R-project.org/package=roxygen2 